\title{
As políticas de inovação para a indústria farmacêutica indiana pós 2005: uma análise sob a abordagem sistêmica da inovação
}

Daniela Falcão (GEI/IE/UFRJ);

Julia Paranhos (GEI/IE/UFRJ)

\section{Resumo:}

Este artigo identifica e analisa, a partir da abordagem sistêmica da inovação, as políticas e instrumentos implementados pela Índia para estimular a capacidade produtiva e tecnológica da sua indústria farmacêutica a partir de 2005, após o período de harmonização ao Acordo TRIPS. O método utilizado foi pesquisa documental das políticas, identificadas pela revisão da literatura, que mais incidem sobre a capacidade produtiva e tecnológica da indústria farmacêutica indiana, a saber: as políticas industrial e de ciência tecnologia e inovação, regulatória de preço e sanitária e de propriedade intelectual. Observouse que estas políticas buscam fortalecer as capacidades tecnológicas domésticas, resguardar a produção nacional e oferecer um ambiente doméstico favorável para os atores privados investirem em inovação. Com base na análise do desenho das políticas, argumenta-se que elas apresentam características de políticas de inovação tal como definidas pela abordagem sistêmica da inovação e funcionam de forma bastante articulada entre si. Dada essas características, o caso indiano serve de aprendizado para outros países em desenvolvimento.

Palavras-chave: inovação; indústria farmacêutica indiana; políticas de inovação.

Códligo JEL: O25; 034; 038.

Área Temática: 6.2 Políticas de Ciência, Tecnologia e Inovação. 


\section{Introdução}

Nos últimos 50 anos, a indústria farmacêutica indiana (IFI) evoluiu de quase inexistente para uma líder mundial na produção de medicamentos genéricos de alta qualidade e baixo custo. Atualmente, a Índia é a $3^{\circ}$ maior fabricante de produtos farmacêuticos no mundo, em termos de volume; a maior exportadora de medicamentos genéricos, respondendo por cerca de $20 \%$ das exportações globais, em volume; fornece mais de $50 \%$ da demanda mundial de muitas vacinas e $60 \%$ dos medicamentos antirretrovirais globais (MANI, 2006; OPPI, 2018; MAKE IN INDIA, s.d). Além disso, a importância da Índia é crescente, o país é o destino preferido de terceirização ${ }^{l}$ no setor farmacêutico devido à alta capacidade produtiva e tecnológica que as empresas farmacêuticas indianas apresentam, aos fortes investimentos governamentais em infraestrutura de $\mathrm{P} \& \mathrm{D}$ e nos baixos custos produtivos, o que torna o processo de desenvolvimento e produção de medicamentos no país mais barato (RUIZ; PARANHOS, 2012). Em 2018, 8 das 20 principais empresas genéricas do mundo eram da Índia; o país possuía em torno de 3.000 empresas farmacêuticas, mais de 10.500 unidades de fabricação e mais de 60.000 marcas genéricas existentes em 60 categorias terapêuticas (MAKE IN INDIA, s.d). Gradualmente, tem-se observado uma transição e aprimoramento produtivo na IFI: de produção de genéricos simples para segmentos de alta barreira de entrada, como genéricos complexos, biossimilares e medicamentos essenciais (OPPI, 2018).

Em termos de valor de mercado, o setor farmacêutico da Índia foi avaliado em US\$ 33 bilhões em 2017 e suas exportações ${ }^{2}$ atingiram US\$ 19,14 bilhões em 2018 e incluem medicamentos a granel, produtos intermediários, formulações de medicamentos, produtos biológicos, produtos à base de plantas e produtos medicinais e cirúrgicos, sendo os EUA o seu principal mercado (IBEF, 2019). Em relação à $\mathrm{P} \& \mathrm{D}$, o investimento (como \% das vendas) das empresas farmacêuticas indianas aumentou de 5,3\% em 2012 para 8,5\% em 2018. Além disso, o setor atraiu ingressos acumulados de investimento estrangeiro no valor de US\$ 15,98 bilhões entre abril de 2000 e março de 2019, segundo dados divulgados pelo Departamento de Política e Promoção Industrial (DIPP) (IBEF, 2019).

Para alcançar tal patamar e prestígio internacional e firmar-se como uma das mais proeminentes produtoras de medicamentos à nível mundial a IFI percorreu um longo caminho. O processo de consolidação e ascensão internacional da indústria farmacêutica na Índia envolveu a combinação simultânea de incentivos e políticas governamentais, mudanças no regime regulatório internacional e estratégias empresariais (RADAELLI; PARANHOS, 2013). A partir da década de 1970, presenciou-se a implementação de diversas políticas industriais, de regulação de preços e de propriedade intelectual com o propósito de garantir a autossuficiência na produção nacional, com vistas a diminuir a dependência estrangeira; fortalecer a indústria farmacêutica doméstica e garantir a disponibilidade de medicamentos a preços acessíveis no país (KALE; LITTLE, 2007). Como resultado, criou-se um ambiente institucional favorável às empresas indianas adquirirem capacidades produtivas e tecnológicas que as permitiram produzir medicamentos genéricos de alto desempenho e tornarem-se suficientemente competitivas para atuar em grandes mercados (Ibidem).

No entanto, a negociação em 1994, de um novo acordo internacional para regular questões relacionadas à propriedade intelectual (PI) - o Acordo TRIPS ${ }^{3}$ - marcou o ponto de virada na estratégia de desenvolvimento da IFI. Tal Acordo, entre outras disposições, estabeleceu um padrão mínimo e obrigatório de proteção à PI extensivo a todos os setores, incluindo o farmacêutico. A obrigatoriedade de concessão de patentes para produtos e processos farmacêuticos por um período de 20 anos, passou a ser uma novidade para muitos países em desenvolvimento (PED). No caso da Índia em particular, não havia concessão de patentes para produtos, apenas para processos farmacêuticos e por um período de sete anos, o que permitia ampla produção de medicamentos genéricos e sua comercialização mais rápida no mercado (RAY, 2008).

\footnotetext{
${ }^{1}$ Outsourcing ou terceirização, em português, consiste na subcontratação de empresas independentes tanto nos mercados nacionais quanto nos internacionais (RADAELLI, 2006).

${ }^{2}$ Vale lembrar que a produção farmacêutica indiana é em grande medida voltada para o mercado externo. Esse grande foco indiano para a exportação foi, em grande parte, estimulado pela crescente desverticalização do processo de produção na indústria farmacêutica, no qual, as grandes farmacêuticas multinacionais passaram a investir mais na etapa final da produção e comprar insumos farmacêuticos ativos (IFAs) e produtos intermediários de países asiáticos, nomeadamente China e Índia, devido aos seus custos produtivos menores.

${ }^{3}$ O Acordo sobre os Aspectos dos Direitos de Propriedade Intelectual Relacionados ao Comércio - TRIPS, em português).
} 
Para os PED, o TRIPS estabeleceu algumas flexibilidades, entre elas um período de transição de dez anos (1995-205) para estes adequarem suas legislações nacionais. A Índia, ao contrário do Brasil, utilizou todo esse período de transição e buscou fortalecer suas capacidades produtivas e minimizar os efeitos adversos do TRIPS na sua produção farmacêutica nacional. Entre as estratégias adotadas neste período destacam-se a presença de maiores investimentos em P\&D inovativa e alianças e parcerias colaborativas com multinacionais para desenvolver novas competências, principalmente, em novas entidades químicas (KALE, 2012; CHATUVERDI, 2011). Assim, em 2005, quando o Acordo TRIPS entrou em vigor no país, as empresas farmacêuticas nacionais estavam melhor preparadas às novas medidas regulatórias.

Dado este contexto, o objetivo geral deste artigo é analisar, sob a perspectiva da abordagem sistêmica de inovação, as políticas e instrumentos implementados pelo governo da Índia para estimular a capacidade produtiva e tecnológica da sua indústria farmacêutica a partir de 2005, após o período de harmonização ao Acordo TRIPS, que trouxe importantes implicações para essa indústria. A escolha deste marco temporal dá-se pelo fato do ineditismo de se analisar e discutir o período pós 2005 na IFI sob a perspectiva das políticas de inovação. Além disso, o período anterior ao TRIPS já está bastante documentado na literatura (MANI, 2006; KALE; LITTLE, 2007; RAY, 2008; TORRES; HASENCLEVER, 2017). O artigo procura demonstrar que a implementação de políticas de inovação com características sistêmicas na IFI é um fator que contribui para tornar o país um grande centro produtivo farmacêutico de referência mundial, como os dados acima apontam.

Para tanto, o método utilizado foi pesquisa documental das políticas, identificadas pela revisão da literatura, que mais incidem sobre a capacidade produtiva e tecnológica da indústria farmacêutica indiana, a saber: as políticas industrial e de ciência tecnologia e inovação, regulatória de preço, sanitária e de propriedade intelectual.

Em seguida, a análise de tais políticas foi realizada com base no referencial teórico da literatura de políticas de inovação (CHAMINADE; EDQUIST, 2006; LUNDVALL; BORRÁS, 2005; BORRÁS; EQUIST, 2013; EDLER; FAGERBERG, 2017). Buscou-se identificar que atividades e instrumentos das políticas de inovação foram desenvolvidas pela Índia; que problemas sistêmicos do processo de inovação (investimento, transição, aprisionamento (lock in), institucionais, e de capacidade e aprendizagem) elas procuraram solucionar; e como articularam e coordenaram as atividades e instrumentos existentes para superarem a presença desses problemas e promoveram a inovação.

Este artigo é dividido em três seções além desta Introdução. A primeira seção discute a importância de políticas de inovação para o desenvolvimento de um sistema de inovação, destacando o papel das políticas explícitas e implícitas nesse cenário. A segunda seção apresenta as políticas e instrumentos - de CT\&I e regulatória de preço, sanitária e PI - da Îndia para a indústria farmacêutica pós 2005. Por sua vez, a terceira seção faz uma análise crítica das políticas e instrumentos identificados à luz da abordagem de políticas sistêmicas de inovação. Por fim, são apresentadas as considerações finais.

\section{O papel das políticas sistêmicas de inovação}

A partir de meados da década de 1990, o termo "política de inovação" se tornou popular entre os formuladores de políticas. Seu surgimento como campo de política está relacionado à crescente ênfase na inovação como uma importante fonte de prosperidade e bem-estar econômico e como uma maneira de lidar com grandes desafios sistêmicos. Em linhas gerais, a literatura que se debruça sobre o tema (CHAMINADE; EDQUIST, 2006; 2010; LUNDVALL; BORRÁS, 2005; BORRÁS; EDQUIST, 2013) define que as políticas de inovação abrangem todas as políticas que apresentam um impacto significativo na atividade de inovação, ou seja, caracterizam-se por influenciar direta e indiretamente os processos inovativos.

Os objetivos e as motivações por detrás da elaboração de políticas de inovação - caracterizado puramente como um processo político - são formulados de forma específica em relação à situação atual do país e/ou em comparação com outros países. Muitas vezes tais políticas apresentam motivações econômicas, como a busca por crescimento econômico, crescimento da produtividade, aumento do emprego e competitividade no mercado internacional. No entanto, as motivações também podem ser de natureza não econômica, como cultural, social, ambiental ou militar/de segurança e prestígio nacional, como era observado durante as duas guerras mundiais e na guerra fria (CHAMINADE; EDQUIST, 2010). 
Compreender a inovação como um processo interativo e sistêmico tem implicações importantes para a concepção e implementação de qualquer tipo de política de apoio à inovação. Na ótica da abordagem de sistemas de inovação, a política de inovação tem como finalidade central promover a interação virtuosa entre os componentes do sistema, de forma a criar um ambiente favorável à geração de conhecimento, ao aprendizado tecnológico, e, consequentemente, promover a inovação (CHAMINADE; EDQUIST, 2010; LUNDVALL; BORRÁS, 2005).

Nos processos de inovação, enquanto a inovação incremental é normalmente realizada pelas empresas, mudanças tecnológicas radicais raramente ocorrem sem intervenção pública. Nesse cenário, uma questão importante para o desenho de políticas de inovação é em quais situações as organizações públicas deveriam intervir e quando não deveriam. Ao contrário da abordagem neoclássica, na abordagem sistêmica da inovação, a discussão sobre a racionalidade para a intervenção política baseiase na presença de problemas sistêmicos e não em falhas de mercado ${ }^{4}$.

As implicações políticas gerais da abordagem sistêmica da inovação são diferentes das da teoria econômica padrão muito pelo fato de que as características das duas estruturas são muito diferentes. A abordagem de sistema de inovação desloca o foco das ações no nível de unidades individuais e isoladas dentro da economia (empresas, consumidores) para as bases coletivas da inovação. Aborda o sistema geral que cria e distribui conhecimento, em vez de seus componentes individuais, e as inovações são vistas como o resultado de processos interativos entre os atores dos sistemas - as empresas não inovam isoladamente, mas com interações contínuas com os outros atores do sistema (nos níveis regional, setorial, nacional e supranacional) (LUNDVALL, 2007).

Nesse sentido, para a abordagem sistêmica, a justificativa básica para atuação do Estado seria a ocorrência de problemas sistêmicos causados, em grande medida, pelo alto grau de incerteza e risco que caracterizam o processo de inovação. Entre os problemas sistêmicos Chaminade e Edquist (2006) destacam: problemas de investimento, de transição, de aprisionamento, institucionais hard e soft e de capacidade e aprendizagem. Esses problemas sistêmicos são apresentados no Quadro 1 a seguir.

\section{Quadro 1 - Problemas sistêmicos do processo inovativo.}

\section{Problemas de investimento}

Necessidade de provisão de infraestrutura, incluindo a infraestrutura física (transporte etc.), a infraestrutura científica (universidades de alta qualidade e laboratórios de pesquisa, institutos técnicos etc.) e a infraestrutura de rede (TI, telecomunicações).

Problemas de transição

Dificuldades que podem surgir quando empresas e outros atores encontram problemas tecnológicos ou enfrentam mudanças nos paradigmas tecnológicos predominantes que excedem suas capacidades atuais. Em muitos casos, as empresas podem não ser capazes de prever o surgimento de novos paradigmas, a difusão de novas tecnologias ou mudanças significativas nos mercados que exigem novas soluções tecnológicas. Além disso, esse cenário envolve um alto grau de incerteza que pode impedir que os atores privados invistam em um novo campo tecnológico.

Problemas de aprisionamento (lock-in)

\footnotetext{
${ }^{4}$ Para a economia neoclássica, uma condição necessária para a intervenção de políticas públicas seria a presença de falhas de mercado. A falha de mercado pode ter causas diversas, mas as mais frequentemente levantadas no contexto da política de inovação são a falta de incentivos para investir na produção de conhecimento. Isto porque o conhecimento tende a ser visto como um bem público não rival e não exclusivo, sendo, portanto, difícil excluir terceiros de se apropriarem de seus resultados. Assim, as firmas racionais, de acordo com esse raciocínio, tendem a ficar longe de tais investimentos, mesmo que os benefícios potenciais para a sociedade como um todo possam ser muito grandes. Neste caso, um mercado autorregulado falharia em assegurar uma alocação socialmente ótima de recursos para a inovação. Para esses economistas, essa "falha de mercado" fornece uma justificativa para intervenções no mercado - ou instrumentos de política - visando aumentar os investimentos em ciência na economia em direção ao nível social "ótimo". Tais intervenções podem assumir diferentes formas, como financiar universidades e institutos de pesquisa, subsidiar pesquisas em empresas privadas e mudar as regras do jogo, fortalecendo, por exemplo, os direitos de propriedade intelectual (FAGERBERG, 2016).
} 
Derivados da inércia socio tecnológica, que pode dificultar o surgimento e disseminação de tecnologias mais eficientes. Esse problema pode ocasionar problemas de transição, na medida em que o foco excessivo em tecnologias existentes pode impedir que as empresas prevejam o surgimento de novas oportunidades tecnológicas.

\section{Problemas institucionais hard e soft}

Ligados a regras formais (regulamentos, leis) - problemas hard, e a regras mais informais e tácitas (cultura social e política, por exemplo) - problemas soft.

\section{Problemas de capacidade e aprendizagem}

Referem-se às competências insuficientes das empresas (humanas, organizacionais, tecnológicas e assim por diante) que podem limitar sua capacidade de aprender, adotar ou produzir novas tecnologias ao longo do tempo. Em outras palavras, o sistema pode ter a infraestrutura e a estrutura institucional certas, mas as organizações do sistema podem ter dificuldades em acessar ou criar novos conhecimentos ou em transformar conhecimento em inovações.

Fonte: Adaptado de Chaminade e Edquist 2006, p.9.

A presença de tais problemas sistêmicos levanta duas questões importantes. Primeiro, um contexto no qual as empresas e os mercados têm desempenho menos eficiente é em relação às atividades em que a incerteza e o risco são grandes. Assim, duas maneiras para o Estado lidar com a incerteza associada a qualquer processo de inovação seria i) fornecendo as informações de que os diferentes atores precisam para definir suas estratégias e ii) fornecendo aos atores os instrumentos, instalações e ambientes para o seu processo de aprendizagem. Isso inclui a oferta de mercados para novos produtos (via contratos e compras públicas, por exemplo) ou a concessão de incentivos à investigação em determinadas áreas prioritárias (incentivos à P\&D) (CHAMINADE; EDQUIST, 2010).

Em segundo lugar, a intervenção política precisa ser seletiva, concentrando-se em produtos, atividades, tecnologias específicas ou setores que atendam melhor aos objetivos (econômicos, sociais, ambientais etc.) do governo (Ibidem). Nesse ponto cabe ressaltar que um dos principais resultados do arcabouço de sistemas de inovação é que os fatores que influenciam a inovação variam de acordo com o setor da economia, ou seja, um mesmo instrumento de política pode apresentar resultados muito diferentes entre os vários setores (MALERBA, 2002). Dessa forma, reconhece-se que a estrutura institucional difere entre as economias nacionais e que isso tem implicações para os tipos de tecnologias e setores que prosperam no contexto nacional. Portanto, conceber uma política de inovação adequada requer conhecimentos específicos sobre as características institucionais do sistema nacional (LUNDVALL; BORRÁS, 2005).

Para tentar solucionar esses problemas sistêmicos presentes no processos de inovação, a literatura (CHAMINADE; EDQUIST, 2006; FAGERBERG, 2016; EDLER; FAGERBERG, 2017; BORRÁS; EDQUIST, 2013, EDQUIST, 2011) descreve uma série de dez atividades do sistema de inovação, não exaustivas, agrupadas em quatro categorias temáticas: (i) provisão de insumos de conhecimento para o processo de inovação; (ii) atividades do lado da demanda; (iii) provisão de elementos constituintes do sistema; e (iv) serviços de suporte para empresas inovadoras. Na verdade, os problemas a serem mitigados pela política de inovação estão relacionados à identificação de deficiências ou gargalos relacionados à essas atividades.

Em primeiro lugar, está a provisão de insumos de conhecimento para o processo de inovação, na qual as políticas públicas podem voltar-se para a criação de conhecimento, através de fornecimento de resultados de $\mathrm{P} \& \mathrm{D}$, e para a construção de competências e qualificação de recursos humanos, seja através de aprendizagem individual (educação e treinamento da força de trabalho para atividades de inovação e P\&D) e/ou aprendizagem organizacional formal e informal. A atuação do Estado, nesse contexto, teria como objetivo diluir os riscos dos investimentos em $\mathrm{P} \& \mathrm{D}$, estimulando o compartilhamento do esforço científico e tecnológico entre instituições públicas e empresas privadas.

A segunda atividade diz respeito às atividades sob a ótica da demanda, como a criação e o fortalecimento de mercados, tanto por meio do mecanismo de compras públicas, intervenções e subsídios públicos quanto apoiando a segurança jurídica ou a formação de confiança e a articulação de requisitos de qualidade como a padronização de especificações técnicas e regulação nos campos econômicos, de saúde e meio ambiente, por exemplo. 
A terceira atividade relaciona-se a formação do ambiente de inovação, que envolveria políticas públicas para estimular o empreendedorismo e à formação de redes para o aprendizado interativo, além de orientar e formular as regras do jogo que facilitarão os processos de inovação, ou seja, é importante, do ponto de vista da política, estudar se as instituições existentes são apropriadas para promover a inovação e fazer a mesma pergunta sobre como as instituições devem ser modificadas ou reconstruídas para induzir a inovação - a evolução e o desenho de novas instituições foram muito importantes nas estratégias de desenvolvimento bem-sucedidas das economias asiáticas, como Coreia e Japão. Por fim, o quarto tipo diz respeito à oferta de serviços de apoio para empresas inovadoras, como financiamento de processos de inovação, apoio à gestão de empresas como acesso a instalações, apoio administrativo para esforços inovadores e prestação de serviços de consultoria.

A compreensão da dinâmica de cada uma dessas atividades pode ser um ponto de partida útil para identificar o papel do governo em estimular o sistema de inovação, mas é preciso levar em consideração os componentes do sistema de inovação e as relações entre eles: organizações ou indivíduos realizam as atividades e as instituições fornecem incentivos e obstáculos que influenciam essas atividades (CHAMINADE; EDQUIST, 2006).

As diferentes atividades do sistema de inovação estão intimamente relacionadas a diversidade de ferramentas para seu estímulo, conhecidos como instrumentos de políticas. De modo geral, existem três grandes categorias de instrumentos usados em políticas públicas: (1) instrumentos regulatórios, (2) instrumentos econômico-financeiros e (3) instrumentos soft (BORRÁS; EDQUIST, 2013). A escolha desses instrumentos é uma decisão crucial para a formulação de uma política de inovação e seus resultados.

Os instrumentos regulatórios utilizam ferramentas legais para a regulação das interações sociais e de mercado, sendo comum serem de natureza obrigatória. Do ponto de vista da política de inovação, os instrumentos regulatórios são regras do jogo para processos de conhecimento e inovação e constituem uma parte importante da estrutura institucional de um sistema de inovação. Exemplos são a regulamentação dos direitos de propriedade intelectual, de organizações de pesquisa e ensino superior, como universidades e organizações públicas de pesquisa, regulamentos de política de concorrência (antitruste) relativos à $\mathrm{P} \& \mathrm{D}$ e atividades inovadoras de empresas no mercado. A relação entre instrumentos regulatórios e inovação pode ser direta, ou seja, projetados com o objetivo explícito de afetar positivamente o conhecimento e as atividades inovadoras, ou indireta quando o objetivo final de um instrumento regulatório específico não é promover a inovação, mas isso acontece de maneira indireta (BORRÁS; EDQUIST, 2013).

Os instrumentos econômico-financeiros fornecem incentivos ou desincentivos às atividades sociais e econômicas específicas. Um dos instrumentos mais amplamente utilizados é o apoio público às organizações de pesquisa, principalmente universidades públicas e organizações públicas de pesquisa. Outros instrumentos fundamentais que usam incentivos econômicos são financiamento competitivo para pesquisa (pesquisa industrial ou básica), incentivos fiscais para $\mathrm{P} \& \mathrm{D}$ realizados no nível da empresa, apoio à transferência de tecnologia e apoio ao capital de risco (Ibidem).

Os instrumentos soft, por sua vez, são caracterizados como sendo não coercitivos e têm sido cada vez mais utilizados na política de inovação nas últimas duas décadas e são em grande parte um complemento aos instrumentos regulatórios e econômicos, constituindo novas abordagens da ação pública em termos de inovação. Exemplos desses instrumentos são códigos de conduta, acordos voluntários e relações contratuais, e parcerias públicas e privadas, que compartilham custos, benefícios e riscos no fornecimento de bens públicos específicos. Esses instrumentos são muito diversos, mas geralmente baseados em persuasão, no intercâmbio mútuo de informações entre os atores e em formas menos hierárquicas de cooperação entre o público e os atores privados (Ibidem).

Borrás e Edquist (2013) salientam a importância de relacionar os diferentes tipos de instrumentos com as diferentes atividades do sistema de inovação e como os diversos instrumentos são utilizados de forma simultânea e combinada. Segundo Mohnen (2005 apud BORRÁS; EDQUIST 2013, p. 22, tradução livre) os instrumentos devem ser combinados entre si pois "a solução de problemas específicos requer abordagens complementares aos aspectos multidimensionais dos problemas relacionados à inovação".

Além disso, esses autores ressaltam que a combinação desses instrumentos para abordar os problemas concretos identificados nos processos de inovação é o que torna a política de inovação sistêmica (BORRÁS; EDQUIST, 2013). Na verdade, uma política de inovação sistêmica contempla 
também as articulações entre políticas e instrumentos de diversos domínios diferentes, de apoio direto e indireto (explícitos e implícitos) à inovação (EDLER; FAGERBERG, 2017). Por política e instrumentos explícitos entende-se a política de ciência tecnologia e inovação oficial, expressa em leis, planos, regulamentos e estatutos e ainda através de declarações governamentais; as políticas e instrumentos implícitos, por sua vez, são aqueles que afetam indiretamente as atividades de ciência, tecnologia e inovação (HERRERA, 1972). Exemplos são as políticas macroeconômicas, regulatórias setoriais e de concorrência, instrumentos de política comercial, poder de compra público, entre outros. Para Herrera (1972) as políticas de inovação implícitas são tão ou mais importantes que as políticas explícitas, tendo em vista que tais políticas têm a capacidade de interferir de forma significativa nas estratégias e capacidades produtivas e inovativas dos atores do sistema, inclusive com capacidade de neutralizar as políticas explícitas.

Sobre a necessidade de articulação entre os instrumentos, Fagerberg (2016) ressalta que se em um sistema dinâmico, um fator crítico, complementar, estiver faltando ou não progredir, isso pode bloquear ou desacelerar o crescimento de todo o sistema. Por exemplo, é de pouca ajuda ter conhecimento superior, se a organização não tiver as habilidades necessárias para sua exploração, ou se faltar financiamento ou demanda. Por esse motivo, os processos que as políticas podem influenciar devem ser em grande parte complementares. Concomitantemente, isso sucinta a questão de que o efeito de uma política específica não pode ser avaliado de forma isolada, ou seja, independente de outras políticas relevantes. A perspectiva do sistema de inovação, portanto, leva a uma perspectiva holística da política (FAGERBERG, 2016).

Portanto, uma política de inovação deve ser vista como um sistema de políticas, que reúne políticas tradicionalmente individuais e independentes (política industrial, científica e tecnológica, por exemplo) em uma nova política sistêmica, com novas racionalidades, novos instrumentos e novos órgãos de governança (CHAMINADE; EDQUIST, 2010).

\section{As políticas de inovação para a indústria farmacêutica na Índia}

Após a entrada em vigor do Acordo TRIPS na Índia, em 2005, as ações e diretrizes políticas no país para o setor farmacêutico tiveram como objetivo central promover um ambiente mais inovador na IFI, contornar os desafios presentes no mercado farmacêutico global e alcançar metas mais elevadas de crescimento até 2020: melhorar a participação da IFI no mercado farmacêutico global para 5\%, em termos de valor; alcançar receitas anuais em torno de 100 bilhões de dólares, que as exportações atingissem Rs. 130,000 crores $^{5}$; tornar a P\&D nacional mais competitiva internacionalmente; e tornar a Índia um dos cinco principais centros de inovação farmacêutica do mundo e de descoberta de medicamentos de ponta a ponta (ÍNDIA, 2013b). Essas aspirações são conhecidas como Pharma Vision 2020 e estão sob a alçada do Department of Pharmaceuticals (DoP).

Por meio do $11^{\circ}$ e $12^{\circ}$ planos quinquenais, planos que estabelecem as principais prioridades, objetivos e metas de crescimento a nível nacional e setorial para cada cinco anos no país, formulados desde 1951, o governo destacou a necessidade de i) implementar uma política nacional de inovação que busque fortalecer as capacidades humanas, institucionais e de infraestrutura da indústria farmacêutica nacional e ii) ampliar a participação e ação conjunta de ministérios/departamentos relevantes e stakeholders na construção e execução de estratégias e políticas mais coordenadas para o desenvolvimento do setor (ÍNDIA, 2008; 2013). O Quadro 2 apresenta um resumo das diretrizes apontadas por esses planos para o desenvolvimento da IFI.

Quadro 2 - Diretrizes para o desenvolvimento da IFI pós 2005

\begin{tabular}{|l|l|}
\hline Estímulo às parcerias & $\begin{array}{l}\text { Promover uma maior colaboração entre os institutos de } \mathrm{P} \& \mathrm{D} \text {, universidades e } \\
\text { empresas do setor privado, alavancando seus pontos fortes e estimulando a maior } \\
\text { difusão de conhecimento e maior apoio a iniciativas de desenvolvimento de tecnologia } \\
\text { em estágio inicial e inovadores de base. }\end{array}$ \\
\hline Econômico/Financeira & $\begin{array}{l}\text { Conceder incentivos financeiros e legislativos para atividades de P\&D incluindo } \\
\text { expansão de dedução fiscal para cobrir atividades como custos internacionais de }\end{array}$ \\
\hline
\end{tabular}

\footnotetext{
${ }^{5}$ Nos documentos oficiais do governo da Índia as medidas de valor são descritas como lakhs e crores, no qual 1 lakhs equivale a 100 mil rúpias e 1 crore equivale a 10 milhões de rúpias.
} 
patenteamento e despesas com litígios de patentes, consultores reguladores, serviços terceirizados de P\&D.

Regulatória $\quad$ Capacitar o setor privado para atender aos padrões da OMS de BPF e outros padrões internacionais de fabricação, para as empresas indianas fortalecerem e aumentarem sua presença nos grandes mercados (EUA e União Europeia).

Desenvolvimento de infraestrutura comum na descoberta e desenvolvimento de medicamentos, tais como, fabricação, distribuição, exportação, dispositivos médicos e assim por diante.

Melhorar os processos de fabricação de genéricos e novos insumos farmacêuticos ativos (IFAs).

Revisar o sistema regulatório, tanto para evoluir e racionalizar estruturas regulatórias para medicamentos biossimilares, combinações de medicamentos fixos, ensaios clínicos e desenvolvimento precoce de medicamentos quanto para reduzir os prazos de aprovação de medicamentos no país.

Fonte: Elaboração própria com base nos documentos dos $11^{\circ}$ e $12^{\circ}$ planos quinquenais indianos.

Para alcançar tais metas estabelecidas na Pharma Vision 2020 e implementar uma estrutura de inovação mais afeita a nova realidade, uma série de políticas e instrumentos de cunho industrial e de CT\&I e regulatórios foram formulados. Em relação as política industriais e de CT\&I, em 2005 estava vigente a Política de Ciência e Tecnologia de 2003 que enfatizava a necessidade de garantir sinergia entre a pesquisa científica e a indústria, a necessidade de altos investimentos em $P \& D$ e a integração de setores socioeconômicos com o sistema nacional de P\&D para resolver problemas nacionais (DHAR; SAHA, 2014). Em 2013, entrou em vigor a atual Política de CT\&I englobando tais questões presentes na política antecessora, no entanto, abarcando o paradigma da inovação como foco adicional.

A Política de CT\&I de 2013 reforçou o objetivo de posicionar a Índia entre as cinco principais potências científicas mundiais até 2020. Para isso, priorizou áreas críticas de $P \& D$, entre elas, a descoberta de medicamentos. Uma das principais aspirações impostas na Política foi aumentar o investimento indiano em P\&D de $0,8 \%$ para $2 \%$ e especifica um aumento na contribuição do setor privado como sua principal estratégia para atingir essa meta - a participação do setor privado deve ser em torno de 50\% nos próximos cinco anos ${ }^{6}$ (MINISTRY OF SCIENCE AND TECHNOLOGY, 2013).

Para atrair um maior investimento privado, as principais ferramentas propostas foram: oportunidades igualitárias tanto para o setor público quanto o privado para a obtenção de fundos públicos; permitir participação de múltiplos stakeholders no sistema indiano de $\mathrm{P} \& \mathrm{D}$; maior estímulo à parcerias público privadas (PPP), para tanto, reconheceu a necessidade de estabelecer grandes instalações de P\&D no modo PPP com disposições para a repartição de benefícios; incentivos para a comercialização de inovações e de parcerias com universidades; e também reconheceu o papel dos direitos de PI no estímulo às atividades de $\mathrm{P} \& \mathrm{D}$. Assim, propõe a criação de uma estrutura reguladora $\mathrm{e}$ legal para compartilhamento de direitos de PI entre inventores e investidores e modificações na política de PI para assegurar que os direitos sejam voltados também para o bem social quando apoiada por fundos públicos e para co-compartilhamento dos direitos de PI gerados sob PPP (MINISTRY OF SCIENCE AND TECHNOLOGY, 2013).

Para isso, diversos departamentos ligados a três Ministérios diferentes ${ }^{7}$, criaram vários instrumentos para a promoção desses objetivos, entre eles: incentivos fiscais para P\&D na indústria e nos institutos de pesquisa em termos de gastos incorridos para obter aprovações regulatórias e preenchimento de patentes no exterior e gastos incorridos em ensaios clínicos na Índia, além de programas de isenção de impostos alfandegários e centrais sobre bens específicos incorridos em pesquisa científica (não sendo despesas sob a natureza de custo de qualquer construção e instalação de fábricas) (IBEF, 2019).

\footnotetext{
${ }^{6}$ De maneira geral (sem especificação setorial), o gasto bruto em P\&D, principalmente pelo setor governamental, compreende 54,4\% do governo central, 7,3\% do governo estadual, $4 \%$ do ensino superior, 5,3\% de indústrias do setor público com contribuição do setor privado em torno de 28,9\%. Os gastos com P\&D do setor privado foram significativos em poucos setores, como produtos farmacêuticos, em 27,7\%; transporte a 14\%; tecnologia da informação em 13,6\%, seguida pela defesa (SANDHYA, 2018).

${ }^{7}$ Ministérios da Ciência e Tecnologia (Department of Scientific and Industrial Research - DSIR e Department of Biotechnology - DBT), Ministério do Comércio e Indústria (Department of Industrial Policy \& Promotion - DIPP) e Ministério de Química e Fertilizantes (DoP).
} 
O Scheme for Development of Pharmaceuticals Industry (SDPI) do DoP (s.d) oferece subsídios e apoio financeiro às PMEs farmacêuticas para criação e atualização de centros de pesquisa farmacêutica de acordo com os padrões da OMS de BPF. O Small Business Innovation Research Initiative (SBIRI) do DSIR (2006) busca fortalecer as unidades industriais privadas existentes e parcerias PPP. Já o Make in India, ligado ao DPIIT e DIPP tem como missão estimular a promoção de exportação e atração de investimento estrangeiro no país (MAKE IN INDIA, s.d).

Reconhecendo o papel da propriedade intelectual na inovação, foram implementados também diversos programas voltados para fortalecer a cultura da PI no país, por meio de incentivos e auxílio na transferência de tecnologia patenteada (Patent Acquisition and Collaborative Research and Technology Development- PACE) e suporte legal e financeiro no depósito e comercialização de patentes na Índia, com foco nas startups (Scheme for Facilitating Startups Intellectual Property Protection - SIPP) (DSIR, 2017; DIPP, 2016).

Adicionalmente, evidencia-se a presença de instrumentos voltados para a ampliação da capacidade tecnológica do setor como o Biopharma Mission e a National Biotechnology Development Strategy I e II, que atuam por meio do fortalecimento de infraestruturas compartilhadas para desenvolvimento e validação de produtos; fortalecimento dos recursos humanos por meio de programas de treinamento especializados para profissionais (bolsas de estudo no exterior e nacionais e de pesquisa de longo prazo para pós-doutorados/jovens cientistas); oportunidades de pesquisa em biotecnologia através de parcerias entre empresas/institutos públicos e privados, parcerias globais com organizações governamentais, organizações não-governamentais e fundações de pesquisa filantrópica para projetos multinacionais; e transferência de tecnologia, incubadoras e sistemas de apoio a PMEs e gestão de PI (DBT, 2015; DBT, s.d).

A fim de adquirir posição de liderança em descoberta e desenvolvimento de medicamentos e continuar a se destacar nas formulações, o governo indiano reconheceu que capital humano é peça chave. Assim, criou dez novos National Institute of Pharmaceutical Education e Research (NIPER) ${ }^{8}$, considerados institutos de importância nacional, concebido para fornecer liderança em ciências farmacêuticas e áreas relacionadas. Com atuação independente, os NIPERs possuem como função: nutrir e promover qualidade e excelência na educação e pesquisas farmacêuticas; realizar cursos de mestrado, doutorado e pós-doutorado e pesquisa em educação farmacêutica; desenvolver uma abordagem multidisciplinar na realização de pesquisa e treinamento de mão de obra farmacêutica; e atuar como núcleo de interação entre academia e indústria ao empreender pesquisas patrocinadas e financiadas, bem como projetos de consultoria (DoP, s. d (b)).

No que diz respeito às políticas regulatórias, o principal foco de ação do governo foi a regulação de PI, de preços e sanitária. Reconhecendo a importância da PI na produção nacional, o governo indiano, ao tornar a legislação de patentes compatível com as obrigações do país nos termos do Acordo TRIPS, em janeiro de 2005, inseriu uma disposição na nova Lei de Patentes - a Seção 3(d) - que impôs uma alta barreira para a concessão de patentes farmacêuticas secundárias9. Foi estabelecido critérios mais rigorosos de patenteamento, partindo-se do princípio de que muitas patentes secundárias não são consideradas invenções e, portanto, não são elegíveis para patentes, a menos que os requerentes demonstrem que elas têm maior eficácia ${ }^{10}$. A Seção 3(d), dessa forma, foi uma ferramenta criado pela

\footnotetext{
${ }^{8}$ Cabe salientar que o primeiro NIPER foi estabelecido em 1998, mas a partir de 2007 tal iniciativa passou a ter maior incidência na agenda política (DoP, s.d(b)).

9 Patentes farmacêuticas secundárias se referem às patentes de novas formas de moléculas e medicamentos existentes. Exemplos incluem formas estruturais alternativas de moléculas já conhecidas, fórmulas e composições revistas, ou novos usos médicos (SAMPAT, SHADLEN, 2015).

${ }^{10}$ Como consta na Lei: "não são invenções na adesão desta Lei: a mera descoberta de uma nova forma para uma substância conhecida que não resulte em uma melhoria na eficácia desta substância ou na mera descoberta de qualquer nova propriedade ou nova utilidade de uma substância conhecida ou no mero uso de um processo, máquina ou aparelho conhecidos, a menos que tais procedimentos resultem em um novo produto ou empregue pelo menos um reagente novo. Para efeito desta cláusula, ésteres, éteres, polimorfos, metabólitos, forma pura, tamanho de partícula, os seus isômeros, misturas de isômeros, complexos, combinações e outros derivados da substância conhecida devem ser considerados a mesma substância, a menos que eles mudem significativamente em propriedades no que diz respeito à eficácia” (ÍNDIA, 2005, tradução livre).
} 
Índia para diminuir o modo como o Acordo TRIPS afeta o acesso a medicamentos e a produção de medicamentos genéricos no país.

Outra intervenção política bastante utilizada pela Índia desde a década de 1970 para estimular o acesso é a regulação de preços dos medicamentos, por meio das chamadas DPCOs. Em 2012, a National Pharmaceutical Pricing Policy (NPPP) implementada a partir da DPCO de 2013, colocou mais medicamentos sob controle de preços - de 74 medicamentos essenciais sob controle de preços pela DPCO de 1995 para 348, incluindo medicamentos contra o câncer e HIV. Esta nova Política de Preços passou a regular os preços dos medicamentos através de preços baseados no mercado de formulações, em vez de regular os preços dos medicamentos através do preço baseado no custo dos medicamentos, como era estipulado pela política de 1994. Assim, fixou-se um teto de preços na Lista Nacional de Medicamentos Essenciais com base no preço médio simples de todas as marcas que possuem participação de mercado (com base no volume de negócios anual móvel) igual ou superior a $1 \%$ do volume de negócios total do mercado desse medicamento (DoP, 2012). Adicionalmente, os preços dos medicamentos que não estão sob controle de preços também serão regulados até certo ponto. A política determina que o governo garantirá que seus preços não aumentem em mais de $10 \%$ em um ano (KOHLI, 2012). Para medicamentos usados no tratamento de doenças como câncer e diabetes o preço foi reduzido em cerca de 25\% (RENGANATHAN et al., 2016).

Com a prerrogativa de incentivar o investimento de empresas farmacêuticas, a NPPP de 2012 estabelece que medicamentos patenteados sob o Indian Patents Act de 1970 e que foram feitos como resultado de produtos ou processos endógenos ficam isentos de controle de preços por um período de cinco anos. Além disso, uma formulação envolvendo um novo sistema de entrega desenvolvido por meio de $\mathrm{P} \& \mathrm{D}$ endógena seria elegível para isenção do controle de preços por um período de 5 anos a partir da data de sua aprovação no mercado na Índia (DoP, 2012).

Ademais, empresas intensivas em P\&D estariam aptas à receber benefícios de preço para os medicamentos sob DPCO desde que cumprissem certas condições, dentre as quais: investisse pelo menos 3\% do faturamento anual de vendas em P\&D ou 500 milhões de rúpias por ano (média dos últimos 3 anos), o que for maior em instalações de pesquisa; b) empregar pelo menos 200 cientistas na Índia (mestres ou doutores empregados pelo menos por um ano); c) possuir e operar instalações de fabricação na Índia que tenham sido aprovadas por pelo menos duas agências reguladoras estrangeiras de renome (EUA, Europa, Japão, Canadá, Austrália, Israel, África do Sul, etc.); e d) tenham apresentado pelo menos 10 pedidos de patentes na Índia em pesquisas feitas na Índia. As empresas que cumprirem essas normas seriam elegíveis para o benefício de dedução ponderada em $200 \%$ no imposto de renda, segundo a Lei de imposto de renda de 1961, até 31 de março de 2015 (MANI, 2006).

No que diz respeito à regulação sanitária, nenhum medicamento pode ser importado, fabricado, armazenado, vendido ou distribuído na Índia, a menos que atenda aos padrões de qualidade especificados na Lei sobre Drogas e Cosméticos de 1940. Além disso, a Índia é um dos signatários do esquema de certificação de qualidade dos produtos farmacêuticos da OMS. O certificado de Good Manufacturing Practices (GMP) da OMS é concedido após a inspeção pela Central Drugs Standard Control Organization (CDSCO $)^{11}$ da Índia e pelas Autoridades de Licenciamento do Estado. O CDSCO, para facilitar a realização de negócios no setor e ampliar as exportações de produtos farmacêuticos, aumentou a validade do certificado GMP da OMS de dois para três anos e estabeleceu que o certificado de não objeção da CDSCO não é mais um requisito necessário para exportações de produtos farmacêuticos no país (OPPI, 2018).

Em 2019, o CDSCO publicou a versão final da New Drugs and Clinical Trials Rules (NDCTR), aplicadas a todos os novos medicamentos, novos medicamentos experimentais para uso humano, ensaios clínicos, estudos de bioequivalência e biodisponibilidade e comitês de ética. Essas novas regras substituíram a Parte XA e o Anexo Y das Regras de Medicamentos e Cosméticos de 1945 e cobrem provisões para promover a pesquisa clínica no país, além de tópicos complexos como medicamento

\footnotetext{
${ }^{11}$ A CDSCO é responsável pela aprovação de medicamentos, condução de ensaios clínicos e tem controle sobre a qualidade dos medicamentos importados na Índia.
} 
órfão ${ }^{12}$ e importação de medicamentos ${ }^{13}$ ainda não aprovados na Índia, mas que já foram aprovados para comercialização em seu país de origem.

Sem dúvidas, uma das principais atualizações impostas pela NDCTR, em termos de incentivos para a produção endógena de medicamentos, foi o estabelecimento de novos cronogramas para reduzir o tempo de aprovação da análise de solicitações de ensaios clínicos. Para os ensaios clínicos de medicamentos desenvolvidos fora da Índia e que já foram aprovados e comercializados em outro país, há um limite de 90 dias úteis para a Autoridade Central de Licenciamento (ACL) responder a solicitação. No entanto, se o medicamento for desenvolvido na Índia ou se a P\&D estiver sendo realizada endogenamente e proposto que o medicamento seja fabricado e comercializado na Índia, o prazo diminui para 30 dias úteis. Caso nenhuma comunicação seja recebida da ACL dentro de 90 dias úteis após o recebimento do pedido, a permissão para conduzir um ensaio clínico será considerada concedida para estudos de bioequivalência e biodisponibilidade de novos medicamentos ou novos medicamentos em investigação. A validade das aprovações de ensaios clínicos foi determinada em dois anos para as empresas farmacêuticas iniciarem um estudo, prorrogável por mais um ano (CDSCO, 2019).

\section{As características sistêmicas das políticas de inovação da Índia}

No que diz respeito à definição de políticas de inovação, conceituada como políticas que apresentam implicações significativas na atividade de inovação, mesmo que seu objetivo principal não seja este, é possível argumentar que as políticas industriais, de CT\&I, de PI e regulatória de preço e sanitária implementadas pós 2005 na Índia, se enquadram como políticas de inovação, uma vez que elas contribuem de forma explícita e implícita para criar um ambiente favorável à geração de conhecimento, ao aprendizado tecnológico, e, consequentemente, promover a inovação na IFI.

Analisando as diretrizes de cada política apresentada na seção anterior, observa-se que elas acabam englobando questões relacionadas aos problemas sistêmicos do processo inovativo, ou melhor, iniciativas para mitigar tais problemas, principalmente aqueles relacionados à incerteza e ao risco do processo inovativo. Nesse contexto, o governo indiano atuou em duas frentes principais para estimular o setor privado a investir em atividades inovativas. A primeira delas foi fornecendo aos atores os instrumentos/incentivos financeiros, instalações e ambientes para o seu processo de aprendizagem. A segunda foi criando um ambiente institucional propício ao setor privado da IFI, principalmente em relação à legislação de PI e regulamentações de preços e sanitária. O Quadro 3 identifica quais problemas sistêmicos do processo inovativo as políticas apresentadas neste trabalho procuram solucionar.

Quadro 3: problemas sistêmicos do processo inovativo e as políticas de inovação da IFI

\begin{tabular}{|c|c|c|c|c|c|c|}
\hline \multirow{2}{*}{\multicolumn{2}{|c|}{ Políticas }} & \multicolumn{5}{|c|}{ Problemas Sistêmicos do Processo Inovativo } \\
\hline & & \multirow{2}{*}{$\begin{array}{c}\begin{array}{c}\text { Problemas de } \\
\text { investimento } \\
\mathrm{e}\end{array} \\
\text { infraestrutura }\end{array}$} & \multirow{2}{*}{$\begin{array}{c}\text { Problemas } \\
\text { de } \\
\text { Transição }\end{array}$} & \multirow{2}{*}{$\begin{array}{l}\text { Problemas de } \\
\text { Aprisionamento } \\
\text { (Lock-in) }\end{array}$} & \multirow{2}{*}{$\begin{array}{c}\text { Problemas } \\
\text { institucionais } \\
\text { Hard e Soft }\end{array}$} & \multirow{2}{*}{$\begin{array}{l}\text { Problemas de } \\
\text { capacidade e } \\
\text { aprendizagem }\end{array}$} \\
\hline \multirow{5}{*}{$\begin{array}{c}\text { Políticas e } \\
\text { instrumentos } \\
\text { industriais e } \\
\text { de CT\&I }\end{array}$} & $\begin{array}{l}\text { CT\&I } \\
2013 \\
\end{array}$ & & & & & \\
\hline & SBIRI & $\mathrm{X}$ & $\mathrm{X}$ & $\mathrm{X}$ & & \\
\hline & $\begin{array}{c}\text { Make in } \\
\text { India }\end{array}$ & X & & & & \\
\hline & PACE & $X$ & $X$ & $X$ & & \\
\hline & $\begin{array}{c}\text { NBDS I e } \\
\text { II }\end{array}$ & $X$ & X & X & $X$ & X \\
\hline
\end{tabular}

\footnotetext{
${ }^{12}$ Medicamentos órfãos foram definidos como um medicamento destinado a tratar doenças que afetam não mais de 500 mil pessoas na Índia. Acordou-se para estes medicamentos uma provisão especial para processo acelerado de aprovação, incluindo uma isenção completa de taxa para registro de ensaios clínicos.

${ }^{13}$ Esta disposição isenta a necessidade de realização de ensaios clínicos locais para novos medicamentos a serem importados desde que o medicamento esteja indicado para doenças graves ou doenças de especial relevância para o cenário de saúde na Índia (tuberculose, hepatite C, H1N1, dengue, malária, HIV ou doenças raras), ou para as quais os medicamentos não estão disponíveis ou possuem um custo elevado ou são um medicamento órfão; não forem relatados eventos adversos graves inesperados para o medicamento; e o requerente concorde em realizar um ensaio clínico de Fase IV para estabelecer a segurança e a eficácia desse novo medicamento (CDSCO, 2019).
} 


\begin{tabular}{|c|c|c|c|c|c|c|}
\hline \multirow{7}{*}{} & $\begin{array}{c}\text { Biopharma } \\
\text { Mission }\end{array}$ & $\mathrm{X}$ & $\mathrm{X}$ & $\mathrm{X}$ & & $\mathrm{X}$ \\
\cline { 2 - 7 } & $\mathrm{SIPP}$ & $\mathrm{X}$ & & & & $\mathrm{X}$ \\
\cline { 2 - 7 } & SDPI & $\mathrm{X}$ & & & & $\mathrm{X}$ \\
\cline { 2 - 7 } & NIPERs & $\mathrm{X}$ & & & $\mathrm{X}$ & \\
\hline \multirow{5}{*}{$\begin{array}{c}\text { Políticas } \\
\text { regulatórias }\end{array}$} & $\begin{array}{c}\text { Legislação } \\
\text { de Patentes } \\
2005\end{array}$ & & & & $\mathrm{X}$ & \\
\cline { 2 - 7 } & $\begin{array}{c}\text { NPPP } \\
2012\end{array}$ & & & & $\mathrm{X}$ & \\
\cline { 2 - 7 } & NDCTR & & & & & \\
\hline
\end{tabular}

Fonte: Elaboração própria.

O Quadro 3 mostra que as políticas implementadas pós 2005, no geral, englobam todos os problemas sistêmicos do processo inovativo, com maior destaque para os problemas de investimento e infraestrutura (com nove políticas abarcando tal problema), problemas institucionais (com cinco políticas), os de transição/aprisionamento que, de certa forma, estão relacionados entre si (com cinco políticas), e os de capacidade e aprendizagem (com quatro políticas). Uma hipótese para os problemas de capacidade e aprendizagem aparentemente estarem menos em foco que os demais, é que estes foram prioridade nas políticas do período anterior e, por meio delas, as empresas indianas conseguiram adquirir capacidades produtivas e tecnológicas significativas. No entanto, isto também demonstra como a preocupação com a capacitação da mão de obra teve continuidade nas ações do governo nas diversas fases de desenvolvimento da IFI, ainda que com redução do número de ações específicas.

Percebe-se também que a maioria das políticas compreendem mais de um problema sistêmico em suas iniciativas. Por exemplo, PACE, SBIRI, Biopharma Mission atuam tanto para mitigar problemas de investimento e infraestrutura quanto de transição e institucionais. O mesmo observa-se no NBDS, na Política de CT\&I, embora o enfoque dos problemas possa variar um pouco. Isso pode nos mostrar como as políticas acabam atuando de forma conjunta e coordenada, uma vez que, diversas políticas perpassam pelos mesmos problemas, reforçando assim seus objetivos e efeitos práticos.

Mais do que mostrar que essas políticas lidam com a questão dos problemas sistêmicos do processo inovativo, é interessante destacar como elas atuam para mitigar a presença de tais problemas. Como a literatura da abordagem sistêmica da inovação salienta, os problemas a serem mitigados pelas políticas de inovação estão relacionados à identificação de deficiências ou gargalos relacionados a determinadas atividades chaves do sistema de inovação. Ademais, como salientado na primeira seção, as diferentes atividades do SI estão intimamente relacionadas a diversidade de ferramentas para seu estímulo, conhecidos como instrumentos de políticas. Nesse sentido, cabe também discutir como as políticas para a IFI englobam essas atividades e instrumentos. O Quadro 4 faz uma sistematização da relação entre as políticas da IFI pós 2005 e as atividades chaves do sistema de inovação agrupadas em suas categorias temáticas.

Quadro 4: Políticas de inovação da IFI e sua relação com as atividades do sistema de inovação

\begin{tabular}{|l|c|c|c|c|}
\hline \multirow{2}{*}{ Políticas } & \multicolumn{5}{|c|}{ Atividades chaves do sistema de inovação } \\
\cline { 2 - 6 } & $\begin{array}{l}\text { Fornecimento de insumos } \\
\text { de conhecimento para o } \\
\text { processo de inovação }\end{array}$ & $\begin{array}{l}\text { Atividades do } \begin{array}{l}\text { lado } \\
\text { demanda }\end{array} \\
\text { da }\end{array}$ & $\begin{array}{l}\text { Fornecimento } \\
\text { constituintes de } \\
\text { Sistema de Inovação } \\
\text { do }\end{array}$ & $\begin{array}{l}\text { Serviços } \\
\text { suporte } \\
\text { empresas } \\
\text { inovadoras }\end{array}$ \\
\hline $\begin{array}{l}\text { Política de } \\
\text { CT\&I 2013 }\end{array}$ & $\mathrm{X}$ & & $\mathrm{X}$ & $\mathrm{X}$ \\
\hline SBIRI & & & & $\mathrm{X}$ \\
\hline Make in India & $\mathrm{X}$ & & & $\mathrm{X}$ \\
\hline PACE & $\mathrm{X}$ & & & $\mathrm{X}$ \\
\hline NBDS I e II & $\mathrm{X}$ & & $\mathrm{X}$ & $\mathrm{X}$ \\
\hline $\begin{array}{l}\text { Biopharma } \\
\text { Mission }\end{array}$ & & $\mathrm{X}$ & $\mathrm{X}$ & \\
\hline NIPERs & & & & \\
\hline $\begin{array}{l}\text { Legislação de } \\
\text { patentes 2005 }\end{array}$ & & & & \\
\hline
\end{tabular}




\begin{tabular}{|l|c|c|c|c|}
\hline SIPP & X & & & $X$ \\
\hline NPPP 2012 & & $X$ & $X$ & \\
\hline NDCTR 2019 & & $X$ & $X$ & $X$ \\
\hline SDPI & & & & X \\
\hline
\end{tabular}

Fonte: Elaboração própria.

No caso indiano, percebe-se que as políticas atuaram com grande enfoque na provisão de insumos de conhecimento para o processo de inovação, principalmente, estimulando a construção de competências e qualificação de recursos humanos na IFI, por meio de vários instrumentos como NIPERs, SIPP, a NBDS, Biopharma Mission. A atuação do Estado, nesse contexto, teve como objetivo diluir os riscos dos investimentos em $\mathrm{P} \& \mathrm{D}$, estimulando o compartilhamento do esforço científico e tecnológico entre instituições públicas e empresas privadas.

A provisão de elementos constituintes do sistema de inovação, principalmente atividades relacionadas à mudança de instituições, também foram estimuladas pelas políticas indianas. As regulamentações de PI, e as normas sanitárias e de preços dos medicamentos foram modificados para atender aos novos padrões regulatórios internacionais e manter a IFI competitiva internacionalmente, principalmente no mercado de genéricos. Em relação às atividades sob a ótica da demanda, estas não foram tão estimuladas quanto as demais. Observa-se que as políticas de cunho regulatório se concentraram mais em apoiar a segurança jurídica, formação de confiança e a articulação de requisitos de qualidade amparados em regulamentações internacionais para incentivar as empresas farmacêuticas a se inserirem em novos mercados globais e aumentarem sua posição de mercado.

O quarto tipo de atividades, referente à oferta de serviços de apoio para empresas inovadoras, também está bastante presente nas políticas indianas. Entre elas, atividades de financiamento (SBIRI, isenção de imposto de renda, PACE, Make in India, Biopharma Mission), apoio à gestão de empresas, como atividades de incubação, acesso a instalações e apoio administrativo para esforços inovadores (Políticas de CT\&I, NBDS, SDPI), e prestação de serviços de consultoria relevantes para processos de inovação, por exemplo, transferência de tecnologia, informações comerciais e consultoria jurídica (PACE, SIPP, NBDS).

Em relação aos instrumentos de políticas, nota-se que as políticas de inovação indianas para a indústria farmacêutica acabam utilizando bastante as três categorias de instrumentos de forma simultânea e combinada. O Quadro 5 sintetiza a relação entre os instrumentos e as políticas de inovação indiana.

Quadro 5 - Políticas de inovação da IFI e sua relação com os instrumentos de políticas

\begin{tabular}{|l|c|c|c|}
\hline \multirow{2}{*}{ Políticas } & \multicolumn{3}{c|}{ Instrumentos de Políticas } \\
\cline { 2 - 4 } & Regulatórios & Econômico-financeiros & Soft \\
\hline Políticas de CT\&I 2013 & $\mathrm{X}$ & $\mathrm{X}$ & \\
\hline SBIRI & & $\mathrm{X}$ & $\mathrm{X}$ \\
\hline Make in India & & $\mathrm{X}$ & \\
\hline PACE & & $\mathrm{X}$ & $\mathrm{X}$ \\
\hline NBDS I e II & & $\mathrm{X}$ & $\mathrm{X}$ \\
\hline Biopharma Mission & & & $\mathrm{X}$ \\
\hline NIPERs & $\mathrm{X}$ & & $\mathrm{X}$ \\
\hline Legislação de patentes 2005 & & $\mathrm{X}$ & $\mathrm{X}$ \\
\hline SIPP & $\mathrm{X}$ & $\mathrm{X}$ & \\
\hline NPPP 2012 & $\mathrm{X}$ & $\mathrm{X}$ & \\
\hline NDCTR 2019 & $\mathrm{X}$ & $\mathrm{X}$ & \\
\hline SDPI & & $\mathrm{S}$ & \\
\hline
\end{tabular}

Fonte: Elaboração própria com base na tipologia de Borrás e Edquist (2013)

Observa-se que os instrumentos soft e econômico-financeiros são utilizados diversas vezes e de maneira articulada. Para exemplificar, programas como PACE, SBIRI, Biopharma Mission, NBDS englobam questões relacionadas tanto ao apoio financeiro para treinamento de pessoal, isenções fiscais e subvenção para P\&D e para modernização tecnológica - instrumentos de cunho econômicofinanceiros - quanto estímulos para as PPP e conhecimentos à firmas inovadoras através de programas de consultoria - instrumentos de caráter mais soft. 
As políticas regulatórias, além de trazerem normas que estimulam a busca por atividades inovativas por parte da IFI tendo em vista que precisam se adaptar aos novos padrões obrigatórios impostos, também oferecem estímulos econômico-financeiros ao conferirem benefícios de preços e isenção de imposto para as empresas desde que estas cumprissem certas condições, como investimento em instalações de pesquisa.

A literatura enfatiza que a combinação entre políticas e instrumentos de diversos domínios diferentes, de apoio direto e indireto à inovação é o que torna uma política de inovação sistêmica. Levando isso em consideração, podemos considerar que as políticas de inovação para a IFI possuem um caráter sistêmico, já que seus objetivos e ações são bastante complementares. Ademais, como já salientado, as políticas de inovação indianas apresentam medidas que abarcam mais de um problema sistêmico do processo inovativo, o que pode reforçar este argumento.

Por fim, cabe destacar como as políticas explícitas e implícitas de inovação para a IFI são articuladas e complementares entre si. Como apresentado na primeira seção, as políticas implícitas têm um papel essencial no processo inovativo tendo em vista que elas podem neutralizar as políticas explícitas. No caso da Índia, percebe-se como as políticas regulatórias (caracterizadas como políticas de inovação implícitas) contribuem para estimular as empresas adquirirem novas capacidades e mercados ao impor novos padrões de qualidade, adequados aos padrões internacionais; ao oferecer prazos de testes clínicos menores para empresas com produção doméstica e desconto de preços. Assim, indiretamente, tendem a complementar e reforçar os objetivos e resultados das políticas de inovação explícitas. Mais ainda, pode-se observar que as políticas implícitas possuem maior relevância para o desenvolvimento da IFI do que as políticas explícitas, tendo em vista que apresentam ações e programas mais focados e específicos para o setor farmacêutico do que as explícitas que apresentam diretrizes mais gerais.

A implementação de políticas de inovação sistêmicas se mostra essencial no setor farmacêutico, principalmente em países em desenvolvimento que não possuem uma capacidade produtiva e tecnológica que faça frente às grandes empresas farmacêuticas e resguarde sua produção e mercado doméstico. Devido à alta complexidade tecnológica deste setor, a existência de uma estratégia nacional assertiva, com medidas voltadas para o fortalecimento da indústria, nos maiores diversos domínios, oferece fortes incentivos para o investimento privado e capacitação produtiva e tecnológica no setor, como o caso indiano bem exemplifica.

\section{Conclusão}

Este artigo identificou e descreveu as políticas e instrumentos implementados pela Índia para estimular a capacidade produtiva e tecnológica da sua indústria farmacêutica a partir de 2005, após o período de harmonização ao Acordo TRIPS, e concluiu que as mesmas podem ser consideradas, em grande medida, como políticas sistêmicas de inovação, segundo a abordagem sistêmica da inovação.

As políticas e instrumentos industriais e de CT\&I formuladas pós 2005 buscaram fortalecer uma cultura de inovação no país para estimular o crescimento da IFI, implementando iniciativas para aumentar o investimento em atividades de P\&D para $2 \%$, estimulando uma maior participação do setor privado através de programas de isenções ficais, financiamento para PME, parceiras público privadas e colaborações internacionais, fortalecendo a interação universidade/institutos de pesquisa-empresas, atração de IED, enfatizando questões de direitos de PI e transferência de tecnologia, capacitação de recursos humanos de qualidade e desenvolvimento tecnológico, priorizando determinados segmentos, como biotecnologia e biossimilares. Por sua vez, as políticas regulatórias implementadas foram direcionadas para aumentar o número de medicamentos sob controle de preços para garantir um maior acesso aos medicamentos; tornar os padrões de qualidades ainda mais compatíveis com as normas internacionais e reduzir o tempo de aprovação dos medicamentos; incentivar instalações comuns de produção de medicamentos; favorecer medicamentos genéricos em detrimento dos medicamentos de marca; e impulsionar as exportações e aumentar a presença de mercado e a competitividade das empresas farmacêuticas nacionais.

Percebe-se assim que as políticas analisadas funcionam de forma bastante articulada entre si. Observou-se que diversos programas implementados adotam iniciativas que muitas vezes contemplam as mesmas questões já ressaltadas por outros instrumentos (por exemplo: fortalecer as unidades privadas existentes, a infraestrutura de $\mathrm{P} \& \mathrm{D}$, reforçar o capital humano qualificado, maior interação entre ICTempresas), reforçando assim a execução dos objetivos propostos pela Política de CT\&I de 2013 e metas 
apresentadas nos últimos planos quinquenais, principalmente de tornar a IFI um dos cincos principais centros de inovação farmacêutica do mundo.

Constata-se que o grande enfoque dessas políticas é oferecer estímulos para incentivar a inovação por parte das empresas farmacêuticas do setor privado e não para as do setor público. O setor público que antes de 2005 era voltado também para a produção e bastante presente nas ações governamentais, adquiriu ao longo dos anos a função de centros de pesquisa para interagir no processo inovativo junto ao setor privado (MANI, 2006). As políticas aqui analisadas são caracterizadas fundamentalmente como políticas de incentivo produtivo e inovativo e formuladas para atender a oferta produtiva das empresas, que, por sua vez, é fortemente voltada para o mercado externo.

Essa posição alcançada pela Índia como um grande player global na indústria farmacêutica é, entre outros fatores, decorrente da presença de políticas de inovação com características sistêmicas, que favorecem o desenvolvimento e crescimento produtivo do setor. Como a literatura ressalta bem, políticas de inovação formuladas e implementadas de forma coordenada e articuladas entre si oferecem fortes incentivos e estímulos para organizações e indivíduos inovarem, tendo assim um papel crucial para a manutenção do dinamismo dos sistemas de inovação.

Embora este artigo não tenha apresentado e discutido os resultados efetivos das políticas analisadas, uma vez que o foco recaiu sobre o desenho das mesmas, a caracterização das políticas de inovação indiana para a indústria farmacêutica serve de importante aprendizado para outros PEDs, em especial, no momento de crise internacional devido à pandemia do novo coronavírus em 2020. A crise sanitária escancarou a grande dependência de diversos países, como o Brasil, mas também EUA e países da União Europeia, na produção de medicamentos e farmoquímicos produzidos na Índia e China (JUNQUEIRA, 2020). Porém, no caso do Brasil, a dependência não é só de produtos, mas também tecnológica, tornando a vulnerabilidade ainda maior. Neste sentido, as discussões apresentadas nesse artigo trazem grandes contribuições e elementos para fortalecer o debate sobre a necessidade de retomada das políticas industriais e de CT\&I para o Complexo Industrial da Saúde no Brasil.

\section{Innovation policies for the Indian pharmaceutical industry after 2005: an analysis based on the innovation system approach}

Abstract: This paper identifies and analyzes, based on the innovation system approach, the policies and instruments implemented by India to stimulate the productive and technological capacity of its pharmaceutical industry from 2005, after the period of harmonization to the TRIPS Agreement. The method used was documentary research of the policies, identified by the literature review, which most focus on the productive and technological capacity of the Indian pharmaceutical industry, namely: industrial and science, technology and innovation policies; price, sanitary and property intellectual regulations. It was observed that these policies seek to strengthen domestic technological capacities, safeguard national production and offer a favorable domestic environment for private actors to invest in innovation. Based on the analysis of policy design, it is argued that they have characteristics of innovation policies as defined by the innovation system approach and are quite articulated. Thus, the Indian case presents lessons for other developing countries.

Keywords: innovation; indian pharmaceutical industry; innovation Policies.

\section{Referências bibliográficas}

BORRÁS, S; EDQUIST, C. The choice of innovation policy instruments. Technological forecasting and social change, v. 80, n. 8, p. 1513-1522, 2013.

CDSCO - Central Drugs Standard Control Organisation. New Drugs and Clinical Trials Rules 2019. Nova Deli, 2019. Disponível em: https://cdsco.gov.in/opencms/opencms/system/modules/CDSCO.WEB/elements/download file divisio n.jsp?num id=NDI2MQ==. Acesso: $12 / 11 / 2019$

CHATUVERDI, S. Multinationals and Monopolies. Pharmaceutical Industry in India after TRIPS. Working Paper Series - WPS, n. 685, 2011. 
CHAMINADE, C., EDQUIST, C. From theory to practice: the use of the systems of innovation approach in innovation policy. Innovation, Science and Institutional Change, Oxford University Press, Oxford, 2006.

CHAMINADE, C., EDQUIST, C. Rationales for Public Policy Intervention in the Innovation Process: Systems of Innovation Approach. In: SMITS, R. E.; KUHLMANN, S.; SHAPIRA, P. (Eds.). Innovation policy - theory and practice. An international handbook, 1a. ed. Cheltenham: Edward Elgar, 2010.

DBT - DEPARTMENT OF BIOTECHNOLOGY. Biopharma Mission. S.d. Disponível em: http://dbtindia.gov.in/schemes-programmes/mission-programmes/biopharma-mission. Acesso: $10 / 10 / 2019$.

DBT - DEPARTMENT OF BIOTECHNOLOGY. National Biotechnology Development Strategy 2015-2020: Promoting bioscience research, education and entrepreneurship. 2015.

DoP - DEPARTMENT OF PHARMACEUTICALS. National Pharmaceutical Policy 2012. Nova Deli, 2012.

DoP - DEPARTMENT OF PHARMACEUTICALS. Scheme for Development of Pharmaceuticals Industry. S.d. Disponível em: https://pharmaceuticals.gov.in/schemes. Acesso: 11/11/2019.

DoP - DEPARTMENT OF PHARMACEUTICALS. National Institute of Pharmaceutical Education \& Research (NIPER). S.d (b). Disponível em: https://pharmaceuticals.gov.in/agencies-underdepartment. Acesso: 20/10/2019

DHAR, Biswajit; SAHA, Sabyasachi. An Assessment of India's Innovation Policies. RIS Discussion paper 184, 2014.

DIPP - DEPARTMENT OF INDUSTRIAL POLICY \& PROMOTION. National intellectual property rights policy, Nova Deli, 2016.

DSIR - DEPARTMENT OF SCIENTIFIC AND INDUSTRIAL RESEARCH. Patent acquisition and collaborative research and technology development (pace): guidelines and application format, 2017.

DSIR - DEPARTMENT OF SCIENTIFIC AND INDUSTRIAL RESEARCH. Report of the working group for eleventh five-year plan, 2006.

EDLER, J; FAGERBERG, J. Innovation policy: what, why, and how. Oxford Review of Economic Policy, v. 33, n. 1, p. 2-23, 2017.

EDQUIST, C. Design of innovation policy through diagnostic analysis: identification of systemic problems (or failures). Industrial and corporate change, v. 20, n. 6, p. 1725-1753, 2011.

FAGERBERG, J. Innovation Policy: Rationales, Lessons and Challenges, Journal of Economic Surveys, v. 31, n. 2, p. 497-512, 2017.

HERRERA, A. Los determinantes sociales de la política científica en América Latina. Política científica explícita y política científica implícita. Redes, v. 2, n. 5, p. 117-131, 1995.

IBEF - INDIA BRAND EQUITY FOUNDATION. Indian Pharmaceuticals Industry Analysis. 2019. Disponível em: https://www.ibef.org/industry/indian-pharmaceuticals-industry-analysis-presentation. Acesso: 08/01/2020.

ÍNDIA. The patents (amendment) ACT, 2005. Nova Deli, 2005.

ÍNDIA. Eleventh Five Year Plan (2007-2012): Inclusive Growth - Volume I. 2008 
ÍNDIA. Twelfth five-year plan (2012/2017): Faster, More Inclusive and Sustainable Growth Volume I. 2013

ÍNDIA. Twelfth Five-Year Plan (2012-2017): Economic Sectors - Volume II, Nova Deli, 2013b.

JUNQUEIRA, D. Coronavírus expõe fragilidade das farmacêuticas, que receberam menor investimento da década sob Bolsonaro. 2020 Disponível em:

https://reporterbrasil.org.br/2020/04/coronavirus-expoe-fragilidade-das-farmaceuticas-quereceberam-menor-investimento-da-decada-sob-bolsonaro/. Acesso: 17/07/2020

KALE, D; LITTLE, S. From imitation to innovation: The evolution of innovative R\&D capabilities in the Indian pharmaceutical industry. Technology Analysis and Strategic Management, v. 19, Issue 5, p. 589-609, 2007.

KALE, D. Innovative capability development in the Indian pharmaceutical industry. International Journal of Innovation and Technology Management, v. 9, n. 02, 2012.

KOHLI, S. National Pharmaceutical Policy 2012. 2012. Disponível em:

https://spicyip.com/2012/12/national-pharmaceutical-policy-2012.html Acesso: 11/10/2019.

LUNDVALL, B-A; BORRÁS, S. Science, technology and innovation policy. The Oxford handbook of innovation, p. 599-631, 2005.

LUNDVALL, B-A Innovation System Research: where it came from and where it might go. CAS Seminar, Oslo, December 4, 2007.

MAKE IN INDIA. Pharmaceuticals. S.d. Disponível em:

http://www.makeinindia.com/sector/pharmaceuticals. Acesso: 09/10/2019.

MALERBA, F. Sectoral systems of innovation and production. Research policy, v. 31, n. 2, p. 247264, 2002.

MANI, S. The sectoral system of innovation of Indian pharmaceutical industry. 2006.

MINISTRY OF SCIENCE AND TECHNOLOGY. Science, technology and innovation policy 2013. Nova Deli, 2013.

OPPI - ORGANISATION OF PHARMACEUTICAL PRODUCERS OF INDIA. 52nd Annual Report 201718. 2018.

RADAELLI, V. A Inovação na Indústria Farmacêutica: forças centrípetas e forças centrífugas no processo de internacionalização. Campinas: Unicamp (Dissertação de Mestrado em Política Científica e Tecnológica), 2006.

RADAELLI, V; PARANHOS, J. Caracterização da trajetória de desenvolvimento da indústria farmacêutica na Índia: elementos para aprendizado. Parcerias Estratégicas, v. 18, n. 36, p. 09-32, 2013.

RAY, A. S. Aprendizagem e inovação na indústria farmacêutica indiana: o papel da IFI e outras intervenções políticas. Revista Eletrônica de Comunicação, Informação \& Inovação em Saúde, v. 2, n. 2, 2008.

RENGANATHAN, R et al. Pharmaceutical pricing policy and control: Indian perspective. Asian Journal of Pharmaceutical and clinical research, Vol 9, Issue 6, 2016.

RUIZ, A; PARANHOS, J. O desenvolvimento de competências tecnológica no setor farmacêutico pós-TRIPS: diferenças entre Brasil, Índia e China. In: Encontro Nacional de Economia da ANPEC, 4. Porto de Galinhas - PE, dez/2012. 
SAMPAT, B.N.; SHADLEN, K.C. TRIPS implementation and secondary pharmaceutical patenting in Brazil and India. Studies in Comparative International Development, v. 50, n. 2, p. 228-257, 2015.

SANDHYA, G. D. India's Science, Technology and Innovation Policy: Choices for Course Correction with Lessons Learned from China. STI Policy and Management Journal, v. 3, n. 1, 2018.

TORRES, R. L; HASENCLEVER, L. A evolução institucional das indústrias farmacêuticas indiana e brasileira revisitada. História econômica \& história de empresas, vol. 20, n, 2, p. 375-406, 2017. 